Bull. Austral. Math. Soc.

VOL. 49 (1994) [7-20]

\title{
UNIQUENESS OF LIMIT CYCLES IN POLYNOMIAL SYSTEMS WITH ALGEBRAIC INVARIANTS
}

\author{
ANDRÉ Zegeling AND ROBERT E. KOOIJ
}

\begin{abstract}
The uniqueness of limit cycles is proved for quadratic systems with an invariant parabola and for cubic systems with four real line invariants. Also a new, simple proof is given of the uniqueness of limit cycles occurring in unfoldings of certain vector fields with codimension two singularities.
\end{abstract}

\section{INTRODUCTION}

Part of Hilbert's 16th problem [13] is to find an upper bound for the number of limit cycles appearing in polynomial systems:

$$
\begin{aligned}
& \frac{d x}{d t}=\dot{x}=\sum_{i+j=0}^{n} a_{i j} x^{i} y^{j}, \\
& \frac{d y}{d t}=\dot{y}=\sum_{i+j=0}^{n} b_{i j} x^{i} y^{j}
\end{aligned}
$$

where $(x, y) \in \mathbb{R}^{2}, t \in \mathbb{R}, a_{i j}, b_{i j} \in \mathbb{R}, n \in \mathbb{N}$.

It is known that the existence of algebraic invariants in polynomial systems influences the number of limit cycles. For example, quadratic systems (that is (1.1) with $n=2$ ) with one line invariant can have at most one limit cycle $[3,4,17$, and 8$]$. The usual procedure in these cases is to transform the system into a Liénard equation and apply a uniqueness theorem. This paper follows the same direction for some unsolved cases in polynomial systems. Since the algebraic invariants in our cases can be used to simplify the Liénard equation (Lemmas $2.1,2.2$ ), it gives some evidence why algebraic invariants in polynomial systems might reduce the number of limit cycles.

The paper is organised as follows. In Section 2 four lemmas for Liénard equations are given. The first one helps to simplify a Liénard equation, given a solution (for example an algebraic invariant). The second lemma concerns a class of differential equations, related to Liénard equations, to which all systems studied in this paper

Received 12 January 1993

The authors wish to thank Professor W.A. Coppel for his useful suggestions.

Copyright Clearance Centre, Inc. Serial-fee code: 0004-9729/94 \$A2.00+0.00. 
belong. The third lemma is a known uniqueness theorem for limit cycles due to Zhang Zhifen. The fourth lemma is an immediate consequence of the third lemma applied to a special class of Liénard equations to which all the systems studied in this paper can be transformed. These four lemmas are applied to polynomial systems with algebraic invariants. In Section 3 quadratic systems with an invariant parabola are studied and it is proved that any such system has at most one limit cycle. This finalises the proof of the uniqueness of limit cycles in quadratic systems with algebraic invariants of degree less than three. In Section 4 cubic systems (that is (1.1) with $n=3$ ) with four real line invariants are considered and the uniqueness of the limit cycle in these systems is proved in a similar way to that used in Section 3. This result should be regarded as an extension of some classical results for quadratic systems. It is well-known that a quadratic system with two line invariants has no limit cycles [1] and that a quadratic system with one line invariant has at most one limit cycle. In [11] it was proved that cubic systems with five real line invariants have no limit cycles. The result of Section 4 therefore completes the analogous results of quadratic systems for cubic systems.

Finally in Section 5 a new approach is presented to the unfolding of vector fields with codimension two singularities. This is a much studied problem that deals with polynomial systems with line invariants [12]. A complete solution of the uniqueness of limit cycles was given by [23, 24]. Another, simpler proof was presented in [5], but according to $[25]$, it contains some mistakes. In $[23,24]$ the main tool of investigation was applying perturbation methods to integrable systems. Here we present a new, simple proof using only the four lemmas of Section 2 involving Liénard equations.

\section{FoUR PRELIMINARY LEMMAS}

In order to investigate the systems mentioned in the introduction, we use four lemmas involving generalised Liénard equations. The first lemma is a generalisation of the method applied in [20].

LEMMA 2.1. Let $f(x), g(x)$ be continuous functions on the open interval $\left(r_{1}, r_{2}\right)$ where $r_{1}<0<r_{2}$, and let $y=\psi(x)$ be a given orbit with $\psi(0) \neq 0$ of the Liénard equation:

$$
\begin{aligned}
& \frac{d x}{d t}=y, \\
& \frac{d y}{d t}=-g(x)-f(x) y .
\end{aligned}
$$

Then (2.1) can be transformed into the equivalent generalised Liénard system

$$
\begin{aligned}
& \frac{d x}{d t}=\varphi(y)-\int_{0}^{x} \bar{f}(\tau) d \tau, \\
& \frac{d y}{d t}=\bar{g}(x)
\end{aligned}
$$


where

$$
\begin{aligned}
\int_{0}^{x} \bar{f}(\tau) d \tau & =\frac{\psi(x)-\psi(0)}{\psi(0)}, \\
\bar{g}(x) & =-\frac{f(x)+\psi_{x}(x)}{\psi(0)}=\frac{g(x)}{\psi(0) \psi(x)}, \\
\varphi(y) & =e^{y}-1 .
\end{aligned}
$$

Proof: Let $z=y-\psi(x)$ in (2.1), then the system becomes:

$$
\begin{aligned}
& \frac{d x}{d t}=z+\psi(x), \\
& \frac{d z}{d t}=-\left[f(x)+\psi_{x}(x)\right] z,
\end{aligned}
$$

where we used that $y=\psi(x)$ satisfies (2.1). Setting $z=-\psi(0) e^{u}$ and rescaling time with $d t / d \tau=-1 / \psi(0)$ we obtain (2.2), after restoring the old variables $u \rightarrow y$, $\tau \rightarrow t$.

REMARK 1. We restrict attention to the region $z / \psi(0)<0$, since it contains any periodic orbit surrounding the origin which intersects the $y$-axis between the origin and the orbit $y=\psi(x)$.

REMARK 2. If $\psi(x)$ happens to be a factor of $g(x)$, as will be the case in the next sections, (2.2) shows that $\bar{g}(x)$ will be simpler than $g(x)$ in (2.1).

The systems discussed in this paper need to be transformed to the form of (2.1), after which we can apply Lemma 2.1 to simplify the system. However, it appears that all our systems have the same structure before the transformation to (2.1). It implies that we can make a shortcut in the calculations by starting with this first type of equation, instead of (2.1), and transform immediately to system (2.2). (This transformation was pointed out to us by Coppel [10]):

LEMмA 2.2. Let $f_{0}(x), f_{1}(x), g_{0}(x), g_{1}(x) g_{2}(x)$ be continuously differentiable functions on the open interval $\left(r_{1}, r_{2}\right)$, where $r_{1}<0<r_{2}$ and with $f_{1}(0) \neq 0$, and let $y=\psi(x)$ be a given orbit with $\psi(0) \neq 0$ of the system:

$$
\begin{aligned}
& \frac{d x}{d t}=f_{0}(x)-f_{1}(x) y, \\
& \frac{d y}{d t}=g_{0}(x)+g_{1}(x) y+g_{2}(x) y^{2} .
\end{aligned}
$$

Then (2.4) can be transformed into the equivalent generalised Liénard system:

$$
\begin{aligned}
& \frac{d x}{d t}=\varphi(y)-\int_{0}^{x} \bar{f}(\tau) d \tau, \\
& \frac{d y}{d t}=-\bar{g}(x) .
\end{aligned}
$$


where

$$
\begin{aligned}
& \bar{g}(x)=\left[f_{1}(x)\left(g_{1}(x)+g_{2}(x) \psi(x)+f_{1}(x) \psi^{\prime}(x)\right)+g_{2}(x) f_{0}(x)\right] / f_{1}^{2}(x) \omega(x) \\
& \bar{f}(x)=\left[f_{0}^{\prime}(x) f_{1}(x)-f_{1}^{\prime}(x) f_{0}(x)-f_{1}^{2}(x) \psi^{\prime}(x)+g_{2}(x) f_{0}(x)\right. \\
& \varphi(y)=e^{y}+\gamma, \gamma=\left[-f_{0}(0)+f_{1}(0) \psi(0)\right] / f_{1}(0) \omega(0)
\end{aligned}
$$

and where $\omega(x)$ satisfies:

$$
\omega^{\prime}(x) / \omega(x)=-g_{2}(x) / f_{1}(x)
$$

Proof: Let $y=\psi(x)+\omega(x) e^{x}$ and $d t / d \tau=-1 / f_{1}(x) \omega(x)$ in (2.4). Then the system becomes (2.5), after restoring the old variables $z \rightarrow y, \tau \rightarrow t$.

REMARK 1. In applications one needs to ensure that $z$ is real in the transformation from (2.4) to (2.5). This can be achieved by choosing the sign of $\omega(x)$ appropriately in (2.6). Notice, however, that $\bar{f}(x) / \bar{g}(x)$, which is crucial in the next sections, is independent of the choice of $\omega(x)$ in (2.5). Therefore $\omega(x)$ will not be specified in the Sections 3 and 4; its explicit form is not needed in the application of Lemma 2.4 and it is assumed that its sign is chosen correctly.

REMARK 2. In [16] it was shown how to transform (2.4) into a Liénard equation (2.1). Lemma 2.2 can be regarded as a combination of this transformation, applied to (2.4), followed by the application of Lemma 2.1 .

The third lemma is a modification of a lemma by Zhang Zhifen [21, 22], see also $[7,9,18]$.

LEMмA 2.3. Let $f(x), g(x)$ be continuously differentiable functions on the open interval $\left(r_{1}, r_{2}\right)$, where $r_{1}<0<r_{2}$, and let $\varphi(y)$ be a continuously differentiable function on $\mathbb{R}$ in:

$$
\begin{aligned}
& \frac{d x}{d t}=\varphi(y)-\int_{0}^{x} f(\tau) d \tau \\
& \frac{d y}{d t}=-g(x)
\end{aligned}
$$

such that

$$
\begin{aligned}
& \text { (i) } \frac{d \varphi}{d y}>0 \text {, } \\
& \text { (ii) } x g(x)>0 \text {, for } x \neq 0 \text {, } \\
& \text { (iii) } f(0) \frac{d}{d x}\left(\frac{f(x)}{g(x)}\right)<0 \text {, for } x \neq 0 \text {. }
\end{aligned}
$$


Then system (2.7) has at most one limit cycle which if it exists, is hyperbolic.

Lemma 2.3 is sufficient for the proofs in the next sections, but it appears that all systems under consideration have the same structure in $\varphi(y), f(x), g(x)$. Therefore a shortcut can be made in the proofs by applying a theorem for this specific class of Liénard equations, presented in [15].

Lемма 2.4. Let $g(x)=(p(x)) /(r(x)), f(x)=(q(x)) /(r(x))$ where $p(x), q(x)$ are polynomials of degree two or less, $r(x) \in C^{\infty}, r(x) \neq 0$ on the open interval $\left(r_{1}, r_{2}\right)$, where $r_{1}<0<r_{2}$, and let $\frac{d \varphi}{d y}>0$ in the generalised Liénard system

$$
\begin{aligned}
& \frac{d x}{d t}=\varphi(y)-\int_{0}^{z} f(\tau) d \tau, \\
& \frac{d y}{d t}=-g(x) .
\end{aligned}
$$

Then system (2.8) has at most one limit cycle in the strip $r_{1}<x<r_{2}$ which, if it exists, is hyperbolic.

The proof of Lemma 2.4 uses Lemma 2.3 together with Dulac's criterion. In Section 3 as an example of how this mechanism works, we shall prove the uniqueness of limit cycles using Lemma 2.3 and Dulac functions only, although Lemma 2.4 could be applied.

\section{QUADRATIC SYSTEMS WITH AN INVARIANT PARABOLA}

In this section we discuss the number of limit cycles of quadratic systems with an invariant parabola. The general quadratic system with an invariant parabola, and with a limit cycle, can be brought to one of the following canonical forms [6]:

$$
\begin{aligned}
& \frac{d x}{d t}=(-a-c)\left(y-x^{2}\right)+(a+b x+c y)+x y \\
& \frac{d y}{d t}=-2\left(y-x^{2}\right)+2 x(a+b x+c y)+2 y^{2} \\
& \frac{d x}{d t}=(a-c)\left(y-x^{2}\right)+(a+b x+c y)+x y \\
& \frac{d y}{d t}=2\left(y-x^{2}\right)+2 x(a+b x+c y)+2 y^{2}
\end{aligned}
$$

where $a>0, b, c \in \mathbf{R}$.

In both systems $y=x^{2}$ is the invariant parabola. In (3.1) [(3.2)] the only critical point not lying on the parabola is $(0,1)[(0,-1)]$. The main result of this section is the following theorem:

THEOREM 3.1. A quadratic system with an invariant parabola has at most one limit cycle which if it exists, is hyperbolic. 
REMARK. It was shown in [2] that a quadratic system with an invariant parabola can have a limit cycle; to prove that the limit cycle is necessarily unique has been a wellknown problem.

Since all other cases of quadratic systems with invariant algebraic curves of degree less than three already were dealt with [19], Theorem 3.1 implies:

THEOREM 3.2. A quadratic system with an invariant algebraic curve of degree less than three has at most one limit cycle which, if it exists, is hyperbolic.

ProOf of THEOREM 3.1: We confine ourselves to the proof of the uniqueness of the limit cycle for the system (3.1), since the system (3.2) can be treated in exactly the same way. As mentioned in Section 2, we could apply Lemma 2.4 instead of Lemma 2.3 after transforming (3.1) to a generalised Liénard equation (3.5) and obtain uniqueness of the limit cycle immediately, but we wish to illustrate here why Lemma 2.4 is valid.

The critical point at $(0,1)$ is the only critical point not on the parabola. A necessary condition for having limit cycles is therefore that $(0,1)$ be an antisaddle. The Jacobian of the linearised system at $(0,1)$ needs to be positive:

$$
\sigma=1+a c+b+a^{2}>0 \text {. }
$$

System (3.1) is of the form of system (2.4), with

$$
\begin{aligned}
& f_{0}(x)=a+b x+(a+c) x^{2} \\
& f_{1}(x)=a-x \\
& g_{0}(x)=2 x[a+(b+1) x], \\
& g_{1}(x)=-2+2 c x, \\
& g_{2}(x)=2 .
\end{aligned}
$$

Since $y=x^{2}$ is an orbit of (3.1), according to Lemma 2.2 it can be transformed into the generalised Liénard system:

$$
\begin{aligned}
& \frac{d x}{d t}=e^{y}+\gamma-\int_{0}^{x} \bar{f}(\tau) d \tau=P, \\
& \frac{d y}{d t}=-\bar{g}(x)=Q,
\end{aligned}
$$

with

$$
\begin{aligned}
& \bar{f}(x)=\left[(3 a+c) x^{2}+2(b+a c) x+a(b+3)\right] /\left(1-\frac{x}{a}\right)^{2}(a-x)^{2}, \\
& \bar{g}(x)=2 \sigma x /\left(1-\frac{x}{a}\right)^{2}(a-x)^{2} .
\end{aligned}
$$

Here we have chosen $\omega(x)$ to be $(1-x / a)^{2}$, satisfying (2.6). It is positive, because in (3.1) the critical point $(0,1)$ lies above the parabola $y=x^{2}$. 
Next we show that system (3.5) satisfies the conditions of Lemma 2.3. The interval $\left(r_{1}, r_{2}\right)$ mentioned in the lemma is in this case $(-\infty, a)$, where $a>0$ according to (3.1). Condition (i) is satisfied because $\frac{d \varphi}{d y}=e^{y}>0$. Condition (ii) is also easily verified using $\bar{g}(x)$ in (3.5) and condition (3.3). In order to be able to apply Lemma 2.3 we only have to check condition (iii). A necessary condition for the existence of limit cycles can be found by applying Dulac's criterion [19] to system (3.5) with $B(y)=e^{\lambda y}$, with $\lambda=-(b+c a) / \sigma$,

$$
\operatorname{div}(B P, B Q)=-\left[(c+3 a) x^{2}+a(b+3)\right] B(y) /\left(1-\frac{x}{a}\right)^{2}(a-x)^{2} .
$$

From (3.6) it follows that limit cycles can only exist if

$$
(c+3 a) a(b+3)<0 .
$$

Under this condition the expression $\frac{d}{d x}\left(\frac{\bar{f}(x)}{\bar{g}(x)}\right)$ is of constant sign for $x \neq 0$ :

$$
\frac{d}{d x}\left(\frac{\bar{f}(x)}{\bar{g}(x)}\right)=\frac{(c+3 a) x^{2}-a(b+3)}{2 \sigma x^{2}} .
$$

Since $\bar{f}(0)=(b+3) / a$, it follows from (3.3), (3.7), (3.8) that condition (iii) of Lemma 2.2 is also satisfied for system (3.5). This completes the proof of Theorem 3.1.

\section{CUBIC SYSTEMS WITH FOUR REAL LINE INVARIANTS}

In this section we study the number of limit cycles of cubic systems with four real line invariants. The main result of this section is the following theorem.

ThEOREM 4.1. A cubic system with four real line invariants has at most one limit cycle which, if it exists, is hyperbolic.

In [14] Kooij studied some aspects of cubic systems with four real line invariants. One of the results of [14] is the following lemma.

LEмMA 4.1. If a cubic system with four real line invariants has a limit cycle then either the four real line invariants are formed by two pairs of parallel lines or amongst the four real line invariants exactly one pair is parallel and three lines pass through the same point.

REMARK. In [14] the existence of limit cycles for the cubic systems described in Lemma 4.1 has been shown by means of the Andronov-Hopf bifurcation.

In the sequel we shall prove that the cubic systems described in Lemma 4.1 have at most one limit cycle. Our method of proof is to transform the cubic systems under consideration to a generalised Liénard system. We consider separately the cases identified in Lemma 4.1. 
THEOREM 4.2. A cubic system with four real line invariants that are formed by two pairs of parallel lines has at most one limit cycle which, if it exists, is hyperbolic.

Proof: Without loss of generality we can assume that the cubic system with four real line invariants formed by two pairs of parallel lines has the following form:

$$
\begin{aligned}
& \frac{d x}{d t}=\left(m x^{2}+n x+1\right)(a x+y), \\
& \frac{d y}{d t}=(y+1)(y-\beta)(c x+d y),
\end{aligned}
$$

where $n^{2}-4 m>0, \beta \neq 0$.

In order to be able to apply Lemma 2.2, we first transform (4.1) by setting $v=$ $y /(1+y), d t / d \tau=1 /(1+y)$ :

$$
\begin{aligned}
& \frac{d x}{d t}=a x k(x)+k(x)(1-a x) y, \\
& \frac{d y}{d t}=-\beta c x+[-\beta d+(2 \beta c+c) x] y+(\beta+1)(d-c x) y^{2},
\end{aligned}
$$

with $k(x) \equiv m x^{2}+n x+1$ and where we have restored the old variables $v \rightarrow y, \tau \rightarrow t$.

System (4.2) is of the form of system (2.4), with

$$
\begin{aligned}
& f_{0}(x)=a x k(x), \\
& f_{1}(x)=k(x)(a x-1), \\
& g_{0}(x)=-\beta c x, \\
& g_{1}(x)=-\beta d+(2 \beta+1) c x, \\
& g_{2}(x)=(\beta+1)(d-c x) .
\end{aligned}
$$

The invariant $y=\beta$ in (4.1) has been transformed into the invariant $y=\beta /(\beta+1)$ in (4.2). Lemma 2.2 can be applied to system (4.2), except if $\beta=-1$. However, for $\beta=-1$, the uniqueness of the limit cycle of system (4.1) can be proved by using (4.2) in a different way, which was done in [14]. In the case $\beta \neq-1$, according to Lemma 2.2 system (4.2) can be transformed into:

$$
\begin{aligned}
& \frac{d x}{d t}=e^{y}+\gamma-\int_{0}^{x} \bar{f}(\tau) d \tau, \\
& \frac{d y}{d t}=-\bar{g}(x),
\end{aligned}
$$

with

$$
\begin{aligned}
& \bar{f}(x)=\left[-a(m+c) x^{2}+(a d-\beta c-a n) x+(d \beta-a)\right] / \omega(x)(a x-1)^{2} k(x), \\
& \bar{g}(x)=(a d-c)(\beta+1) x / \omega(x)(a x-1)^{2} k(x),
\end{aligned}
$$


where $\omega(x)$ satisfies $\omega^{\prime}(x) / \omega(x)=(\beta+1)(d-c x) / k(x)(1-a x)$.

Therefore we arrive at a system satisfying the conditions of Lemma 2.4, with

$$
\begin{aligned}
& \varphi(y)=e^{y}+\gamma, \\
& q(x)=-a(m+c) x^{2}+(a d-\beta c-a n) x+d \beta-a, \\
& p(x)=(a d-c)(\beta+1) x, \\
& r(x)=\omega(x)(a x-1)^{2} k(x) .
\end{aligned}
$$

The zeros of $(a x-1)^{2}$ and $k(x)$ determine the interval $\left(r_{1}, r_{2}\right)$ mentioned in Lemma 2.4. The uniqueness of the limit cycle follows immediately and the proof of Theorem 4.2 is completed.

REMARK. The proof of Theorem 4.2 also holds for $n^{2}-4 m<0$ in system (4.1), that is if the cubic system has one real pair of parallel line invariants and one complex conjugated pair of parallel line invariants.

THEOREM 4.3. A cubic system with four real line invariants such that exactly one pair of lines is parallel and three lines pass through the same point, has at most one limit cycle which, if it exists, is hyperbolic.

The proof of Theorem 4.3 basically runs the same as the proof of Theorem 4.2. We just point out one difference. For the cubic system mentioned in Theorem 4.3 the generalised Liénard equation takes the form

$$
\begin{aligned}
& \frac{d x}{d t}=e^{y}+\gamma-\int_{0}^{x} \frac{p_{2}(s)}{h_{1}(s) h_{2}(s) h_{3}(s) \omega(s)} d s, \\
& \frac{d y}{d t}=\frac{q_{2}(x)}{h_{1}(x) h_{2}(x) h_{3}(x) \omega(x)},
\end{aligned}
$$

where $p_{2}(x)$ and $q_{2}(x)$ are quadratic functions in $x$ and $h_{1}(x), h_{2}(x)$ and $h_{3}(x)$ are linear functions. The zeros of $h_{1}(x), h_{2}(x)$ and $h_{3}(x)$ define the interval $\left(r_{1}, r_{2}\right)$ mentioned in Lemma 2.3. The uniqueness of the limit cycle follows from Lemma 2.4 .

Theorem 4.1 follows from Lemma 4.1 and Theorems 4.2 and 4.3.

\section{UNFOLDINGS OF CODIMENSION TWO SINGULARITIES}

In this section we investigate the number of limit cycles in unfoldings of vector fields with codimension two singularities. Without going into the details of the origin of the problem, we just state here that the main problem is to determine the number of limit cycles of the following two systems for the amplitudes [5]:

$$
\begin{aligned}
& \frac{d x}{d t}=\varepsilon_{1} x+B x y+x y^{2}, \\
& \frac{d y}{d t}=\varepsilon_{2}+\eta x^{2}-y^{2},
\end{aligned}
$$


where $x \geqslant 0, \eta= \pm 1, B \neq 0,\left|\varepsilon_{1}\right|,\left|\varepsilon_{2}\right| \ll 1$,

$$
\begin{aligned}
& \frac{d x}{d t}=x[-q+q x+(q+1) y] \\
& \frac{d y}{d t}=y[p-(p+1) x-p y]+\varepsilon_{1} y+\varepsilon_{2} x y+\varepsilon_{3} x^{2} y
\end{aligned}
$$

where $p, q \in \mathbb{R}, q \neq-1, x \geqslant 0, y \geqslant 0,\left|\varepsilon_{1}\right|,\left|\varepsilon_{2}\right|,\left|\varepsilon_{3}\right| \ll 1$,

In [5] also so-called nondegenerate conditions on the parameters were imposed, but since we shall not use them, we shall not list them here to avoid confusion. These two systems occur in the unfolding of doubly degenerate singularities of vector fields in $\mathbb{R}^{n}$, $n \geqslant 2$, of which the linear part takes the form (after reduction to a centre manifold [12]):

$$
\begin{aligned}
A_{1} & =\left[\begin{array}{rrr}
0 & 1 & 0 \\
-1 & 0 & 0 \\
0 & 0 & 0
\end{array}\right] \text { for }(5.1) \\
A_{2} & =\left[\begin{array}{rrrr}
0 & 1 & 0 & 0 \\
-1 & 0 & 0 & 0 \\
0 & 0 & 0 & \omega \\
0 & 0 & -\omega & 0
\end{array}\right], \omega \notin \mathbb{Q}, \text { for }(5.2) .
\end{aligned}
$$

Systems (5.1) and (5.2) were used in [5], with the exception that in (5.2) we rewrote the perturbation terms. This was done with the recurrence relations in appendix $B$ of [5]. For small values of the perturbation parameter $\varepsilon$ this does not influence the number of limit cycles. Our choice makes it easier to transform to a Liénard equation, whereas the system equivalent to (5.2) used in [5] is more difficult to transform.

To study (5.1) and (5.2) we consider the more general systems:

$$
\begin{aligned}
& \frac{d x}{d t}=c_{1} x+c_{2} x y+c_{3} x y^{2} \\
& \frac{d y}{d t}=c_{4}+c_{5} x^{2}+c_{6} y^{2}
\end{aligned}
$$

where $x \geqslant 0, c_{i} \in \mathbb{R}$,

$$
\begin{aligned}
& \frac{d x}{d t}=x\left[d_{1}+d_{2} x+d_{3} y\right] \\
& \frac{d y}{d t}=y\left[d_{4}+d_{5} x+d_{6} y+d_{7} x^{2}\right]
\end{aligned}
$$

where $x \geqslant 0, y \geqslant 0, d_{i} \in \mathbf{R}$.

In the remainder of this section we shall prove: 
TheOrem 5.1. Systems (5.4) and (5.5) have at most one limit cycle which, if it exists, is hyperbolic.

Since (5.1) and (5.2) are subfamilies of (5.4) and (5.5) an immediate consequence of Theorem 5.1 is:

THEOREM 5.2. In the amplitude equations for unfoldings of codimension two singularities of nonresonant vector fields in $\mathbb{R}^{n}$ at most one limit cycle appears and if it appears it is hyperbolic.

REMARK. In $[23,24]$ the existence of limit cycles in systems (5.1) and (5.2) was proved.

Proof of Theorem 5.1: After setting $x^{2}=e^{u}$ in system (5.4) we obtain:

$$
\begin{aligned}
& \frac{d u}{d t}=2 c_{1}+2 c_{2} y+2 c_{3} y^{2}, \\
& \frac{d y}{d t}=c_{4}+c_{5} e^{u}+c_{6} y^{2},
\end{aligned}
$$

which is system (2.8) (changing $u \rightarrow y, y \rightarrow x$ ) with $\varphi(y)=c_{5} e^{y}+c_{4}, p(x)=$ $-\left(2 c_{1}+2 c_{2} x+2 c_{3} x^{2}\right), q(x)=-2 c_{6} x, r(x)=1$. By a reversal of time $t \rightarrow-t, c_{5}$ can be assumed to be positive. The uniqueness of limit cycles follows immediately from Lemma 2.3. System (5.5) is already of the form (2.4) with:

$$
\begin{aligned}
& f_{0}(x)=x\left(d_{1}+d_{2} x\right), \\
& f_{1}(x)=-d_{3} x, \\
& g_{0}(x)=0, \\
& g_{1}(x)=d_{4}+d_{5} x+d_{7} x^{2}, \\
& g_{2}(x)=d_{6} .
\end{aligned}
$$

According to Lemma 2.2, system (5.5) can be transformed into a generalised Liénard system, because it has an invariant $y=0$ :

$$
\begin{aligned}
& \frac{d x}{d t}=e^{y}+\gamma-\int_{0}^{x} \bar{f}(\tau) d \tau, \\
& \frac{d y}{d t}=-\bar{g}(x),
\end{aligned}
$$

with

$$
\begin{aligned}
& \bar{f}(x)=\left[d_{2}\left(d_{3}+d_{6}\right) x+d_{1} d_{6}\right] / d_{3}^{2} x \omega(x), \\
& \bar{g}(x)=\left[-d_{3} d_{7} x^{2}+\left(d_{2} d_{6}-d_{3} d_{5}\right) x+d_{1} d_{6}-d_{3} d_{4}\right] / d_{3}^{2} x \omega(x),
\end{aligned}
$$

where $\omega(x)$ satisfies $\omega^{\prime}(x) / \omega(x)=d_{6} / d_{3} x$.

Finally we make a translation in $x$, such that the singularity (around which the limit cycle should occur) at $x=x_{0}$, where $x_{0}>0$, is moved to $x=0$. This is necessary, 
since in the Lemma 2.4 it is implicitly assumed that the singularity has an $x$-coordinate equal to zero. After the translation $\bar{x}=x-x_{0}$, system (5.8) is still of the same form, but with (omitting the bar):

$$
\begin{aligned}
& \bar{f}(x)=\left[\lambda_{0}+\lambda_{1} x+\lambda_{2} x^{2}\right] / d_{3}^{2}\left(x+x_{0}\right) \omega\left(x+x_{0}\right), \\
& \bar{g}(x)=x\left[\mu_{0}+\mu_{1} x\right] / d_{3}^{2}\left(x+x_{0}\right) \omega\left(x+x_{0}\right),
\end{aligned}
$$

where $\lambda_{0}, \lambda_{1}, \lambda_{2}, \mu_{0}, \mu_{1}, x_{0}$ are functions of the parameters $d_{1}, d_{2}, d_{3}, d_{4}, d_{5}, d_{6}, d_{7}$, and where we have used that $\bar{g}\left(x_{0}\right)=0$ in (5.8).

We do not specify the parameters in (5.9), because in order to apply Lemma 2.4 all we need to know is the structure of $f(x), g(x)$ in the generalised Liénard system. The system (2.4) with the functions given in (5.9), satisfies the conditions of Lemma 2.4, with $\varphi(y)=e^{y}+\gamma, q(x)=\lambda_{0}+\lambda_{1} x+\lambda_{2} x^{2}, p(x)=x\left(\mu_{0}+\mu_{1} x\right), r(x)=$ $d_{3}^{2}\left(x+x_{0}\right) \omega\left(x+x_{0}\right)$. We choose $r_{2}$ to be $-x_{0}$ and $r_{1}$ to be $+\infty$. Then $r(x) \neq 0$ on this interval and the uniqueness of the limit cycle follows immediately.

REMARK. The proof of the uniqueness is an extension of the work done on the Bogdanov-Takens system [12]. For that system the uniqueness of the limit cycle in the unfolding of a codimension two singularity in the plane, was proved originally using Pontryagin-integral techniques [12]. However, similar to what was done in this section, it is much easier to prove this by considering the system to be a part of a larger family of vector fields. Interestingly enough for the Bogdanov-Takens system this result was obtained already in China before the Bogdanov-Takens system itself appeared in the picture [19].

\section{REFERENCES}

[1] N.N. Bautin, 'On periodic solutions of a system of differential equations', (Russian), Prikl. Mat. Mekh. 18 (1954), 128.

[2] Chen Shuping, 'Limit cycles of a real quadratic differential system having a parabola as an integral curve', (Chinese), Kexue Tongbao 30 (1985), 401-405.

[3] L.A. Cherkas and L.I. Zhilevich, 'Some tests for the absence or uniqueness of limit cycles', (Russian), Differentsial'nye Uravneniya 6 (1970), 1170-1178. Translated in Differential Equations 6 (1970), 891-897.

[4] L.A. Cherkas and L.I. Zhilevich, 'The limit cycles of certain differential equations', (Russian), Differentsial'nye Urauneniya 8 (1972), 1207-1213. Translated in Differential Equations 8 (1972), 924-929.

[5] Shui-Nee Chow, Chenghzi Li and Duo Wang, 'Uniqueness of periodic orbits of some vector fields with codimension two singularities', J. Differential Equations 77 (1989), 231-253.

[6] C.J. Christopher, 'Quadratic systems having a parabola as an integral curve', Proceedings of the Royal Society of Edinburgh 112 A (1989), 113-134. 
[7] W.A. Coppel, 'Quadratic systems with a degenerate critical point', Bull. Austral. Math. Soc. 38 (1988), 1-10.

[8] W.A. Coppel, 'Some quadratic systems with at most one limit cycle', Dynamics Reported 2 (1989), 61-88.

[9] W.A. Coppel, 'A new class of quadratic systems', J. Differential Equations 92 (1991), 360-372.

[10] W.A. Coppel, Private communication.

[11] Dai Guoren and Wo Songlin, 'Closed orbits and straight line invariants in $E_{3}$ systems', (Chinese), Acta Math. Sci. (Chinese) 9 (1989), 251-261.

[12] J. Guckenheimer and P. Holmes, Nonlinear oscillations, dynamical systems, and bifurcations of vector fields (Springer-Verlag, Berlin, Heidelberg, New York, 1983).

[13] D. Hilbert, 'Mathematical problems', Mary Newson Transl, Bull. Amer. Math. Soc. 8 (1902), 437-479.

[14] R.E. Kooij, 'Some new properties of cubic systems', report 90-62, (Delft University of Technology, 1990).

[15] R.E. Kooij, 'Existence and uniqueness of limit cycles for a special system of differential equations on the plane', report 92-63, (Delft University of Technology, 1990).

[16] Liu Jun, 'Transformations and their applications in planar quadratic systems', (Chinese), J. Wuhan Iron and Steel College 4 (1979), 10-15.

[17] G.S. Rychkov, 'The limit cycles of the equation $u(x+1) d u=\left(-x+a x^{2}+b x u+c u+\right.$ $\left.d u^{2}\right) d x^{\prime}$, (Russian), Differential'nye Uravneniya 8 (1972), 2257-2259. Translated in Differential Equations 8 (1972), 1748-1750.

[18] Wang Xian, 'On the uniqueness of limit cycles of the system $\dot{x}=\varphi(y)-F(x), \dot{y}=-g(x)$ ', (Chinese), J. Nanjing Univ. Natur. Sci. 26 (1990), 363-372.

[19] Ye Yanqian, Theory of limit cycles, Transl. Math. Monographs 66 (American Mathematical Society, Providence, R.I., 1986).

[20] Zhang Pingguang, 'Study of non-existence of limit cycles around a weak focus of order two or three for quadratic systems', Chinese Science Bull. 35 (1990), 1156-1161.

[21] Zhang Zhifen, 'On the uniqueness of limit cycles of certain equations of nonlinear oscillations', (Russian), Dokl. Akad. Nauk SSSR 119 (1958), 659-662.

[22] Zhang Zhifen, 'Proof of the uniqueness theorem of limit cycles of generalized Liénard equations', Appl. Anal. 23 (1986), 63-76.

[23] H. Zoladek, 'On the versality of a family of symmetric vector fields in the plane', Math. USSR-Sb 48 (1984), 463-492.

[24] H. Zoladek, 'Bifurcations of certain family of planar vector fields tangent to axes', $J$. Differential Equations 67 (1987), 1-55.

[25] H. Zoladek, 'Quadratic systems with center and their perturbations'. Preprint, (University of Warsaw, 1990) . 
Faculty of Technical Mathematics and Informatics

Delft University of Technology

P.O. Box 5031

2600 GA Delft

The Netherlands 